\title{
A GEOMETRIC APPROACH TO CONSTRAINED MOLECULAR DYNAMICS AND FREE ENERGY*
}

\author{
CARSTEN HARTMANN † AND CHRISTOF SCHÜTTE †
}

\begin{abstract}
We consider a molecule constrained to a hypersurface $\Sigma$ in the configuration space $\mathbf{R}^{m}$. In order to derive an expression for the mean force acting along the constrained coordinate we decompose the molecular vector field, and single out the direction of the respective coordinate utilising the structure of affine connections. By these means we reconsider the well-known results derived by Sprik et al. [1] and Darve et al. [2]; we gain concise geometrical insight into the different contributions to the force in terms of molecular potential, mean curvature, and the connection 1form of the normal bundle over the submanifold $\Sigma$. Our approach gives rise to a Hybrid Monte-Carlo based algorithm that can be used to compute the averaged force acting on selected coordinates in the context of thermodynamic free energy statistics.
\end{abstract}

Key words. molecular dynamics, holonomic constraints, free energy, mean force, affine connection, normal connection, mean curvature, metric entropy, hybrid Monte-Carlo, blue moon method.

AMS subject classifications. 37J05, 70F20, 70G10, 65C35

\section{Introduction}

Many simulations in molecular-dynamics (MD) applications are devoted to the calculation of free energy profiles along selected essential coordinates [3]. These coordinates may be slow degrees of freedom or parameterisations of certain pathes along which a reaction takes place. Here, the term "reaction" is understood in a very broad sense, and mostly refers to any conformational transition in a molecule.

Theoretically, the free energy observable could be easily computed from the invariant measure of the underlying dynamical system. However, since, e.g., conformational changes occur rather rarely, reliable sampling of these parts of the phase space measure is a relatively tedious issue $[4,5]$. Thus, one approach amongst others is to constrain the system to fixed values of those coordinates one is interested in, and which correspond to rare events. Then the free energy turns out to be the potential of the mean force which acts along the constraint coordinate. This technique is known as thermodynamic integration, and it goes back to [6]. The hope is that once one has successfully identified the essential coordinates sampling the invariant measure of the remaining free coordinates should be comparably fast.

During the last few years there have been made many attempts to derive expressions for the mean force along specified coordinates computed from constrained MD simulations. Most authors proposed to exploit the force of constraint, that is necessary to conserve the constraint during the course of integration $[1,2,7,8]$. Nevertheless at the beginning, there was some disagreement, firstly, about the correct expression for the force acting on the reaction coordinate as well as, secondly, about the proper averaging procedure - which is the probability measure to be taken? There are even incommensurable definitions of free energy (see the review [9]). Once all these problems have been resolved, it is natural to ask, how the mean force can be evaluated

${ }^{*}$ Received: March 25, 2004. Accepted (in revised version): November 16, 2004. Communicated by Eric Vanden-Eijnden.

${ }^{\dagger}$ Freie Universität Berlin, Institute of Mathematics II, Arnimallee 2-6, 14195 Berlin, Germany (chartman@math.fu-berlin.de). Supported by the DFG Research Center "Mathematics for Key Technologies" (FZT 86) in Berlin.

${ }^{\ddagger}$ Freie Universität Berlin, Institute of Mathematics II, Arnimallee 2-6, 14195 Berlin, Germany (schuette@math.fu-berlin.de). 
in practical applications, or, secondly: how can the desired phase space measure be generated by the constrained dynamics. Although the results presented here are not new, our mathematical elaboration of the free energy literature allows for two key issues: at first it provides a clear and concise interpretation of the notion of mean force along a reaction coordinate, and even more important, it enables us to derive a Hybrid Monte-Carlo (HMC) scheme for constrained MD simulations. With this tool at hand we are then able to compute free energy profiles along a reaction coordinate from a constrained MD trajectory. The main advantage of this novel method is that it is conceptually very simple and lucid, and that ergodicity poses no problem which might be even most important. Additionally it offers control over the numerical error. We shall shortly sketch the approach.

1.1. Lagrangian vector fields and reaction coordinates. Consider a molecular system whose motion is governed by the Lagrangian function $L(q, \dot{q})$ which is as usual kinetic minus potential energy. We introduce a reaction coordinate defining a scalar-valued function $c(q)$ depending upon the position coordinates only. We want to know the following: which is the mean force acting along the reaction coordinate $c$ for a specific value of $c^{\prime}$. To this end we firstly compute the unavenged force, which we shall denote by $f$. In principle this can be done by a change of coordinates according to $\varphi: q \mapsto(x, c)$, where the reader should be aware of our abusive notation using the same symbol for map $c(q)$ and its coordinate counterpart $c$. Pulling back the original Lagrangian by the diffeomorphism $\varphi$ the force is given by the expression

$$
f\left(x, c^{\prime}, \dot{x}, \dot{c}\right)=-\left.\frac{d}{d t} \partial_{\dot{c}}\left(L \circ \varphi^{-1}\right)(x, c, \dot{x}, \dot{c})\right|_{c=c^{\prime}} .
$$

Though this method is available a proper change of coordinates is in many cases a big mess and not very insightful regarding the underlying physics or geometry. Therefore we adopt an alternative approach that uses the decomposition of the vector field $Z$ that is generated by $L$ into its horizontal and vertical components. Here "vertical" labels the direction of the reaction coordinate. In coordinates the vector field $Z$ reads

$$
Z(q, \dot{q})=\nabla_{\dot{q}} \dot{q}+\operatorname{grad} U(q),
$$

where we have set the constant mass to unity. Note that the solutions to the associated Euler-Lagrange equations are the integral curves of $Z$. The symbol $\nabla_{\dot{q}} \dot{q}$ denotes the affine connection or covariant time derivative, respectively, which indicates the acceleration along a curve $q(t) \in \mathbf{R}^{m}$. Concretely it describes how the velocity vector $\dot{q}(t) \in T_{q(t)} \mathbf{R}^{m}$ changes if one follows the direction of a curve $q(t)$, where the direction is specified by the tangent $\dot{q}(t)$ to the curve. Strictly speaking the covariant derivative is explained for vector fields. But since $\nabla_{\dot{q}} \dot{q}$ depends only locally on the values of $\dot{q}(t)$ at $q(t)$, we can consider $\dot{q}$ as a vector field that coincides with $\dot{q}(t)$ at $q(t)$.

Thus given a curve $q(t) \in \mathbf{R}^{m}$ the question is which is the component of $Z$ in the direction of the reaction coordinate $c$ at $c=c^{\prime}$. Recall that a vector field $Z$ can be written in the form $Z=Z^{i} \partial_{i}$. We therefore may ask which is the component of $Z$ in the direction of the vector $\partial_{c} \in \mathbf{R}^{m}$ at $c=c^{\prime}$. Note that the vector $\partial_{c}$ is proportional to the gradient $D_{q} c$, and that the gradient is normal to the fibre $\Sigma=c^{-1}\left(c^{\prime}\right)$. If we let $V_{q}$ denote the orthogonal projection onto the normal direction, we find for $q \in \Sigma$

$$
\partial_{c}=\left\|D_{q} c(q)\right\|^{-1} V_{q} D_{q} \quad \text { with } \quad D_{q}=\left(\partial_{q^{1}}, \ldots, \partial_{q^{m}}\right)^{T} .
$$

This result is known due to [10]. Here it appears rather naturally; the reason is that $D_{q}$ provides the canonical basis for the vector field $Z$, and we can project this basis 
onto the vector $\partial_{c}$. Then the force $f$ is related to $Z$ by

$$
f(q, \dot{q})=\left\|D_{q} c(q)\right\|^{-1} V_{q} Z(q, \dot{q}), \quad q \in \Sigma .
$$

In order to illustrate what is going on consider the hypersphere $S^{m}$ which has codimension 1 in $\mathbf{R}^{m}$ and that can be described globally by the real-valued function $c(q)=1$. Let $q(t)$ be a curve on $S^{m}$ and let the vertical space be the span of the normal vector $D_{q} c \neq 0$. Accordingly, let $H_{q}=\mathbf{1}-V_{q}$ denote the orthogonal projection onto the horizontal space $T S^{m}$. Then our Lagrangian vector $Z$ field admits a decomposition

$$
Z(q, \dot{q})=\nabla_{\dot{q}} H_{q} \dot{q}+\nabla_{\dot{q}} V_{q} \dot{q}+\operatorname{grad} U(q)
$$

where the horizontal velocities $H_{q} \dot{q}$ constitute the dynamics on the sphere, whereas the vertical velocities $V_{q} \dot{q}$ are assigned to the motion normal to $S^{m}$. Now which is the vertical force? Obviously if the curve lies on $S^{m}$ we have $V_{q} \dot{q}=0$ and the vertical part of $Z$ yields essentially the constraint force that is needed to stay on the sphere. However if we take a curve hitting the sphere transversally, we have to bring up a larger force in order to bring back the curve to the sphere. In that case $V_{q} \dot{q}$ will be different from zero, and in general the derivative of the vertical acceleration will contribute to the force.

1.2. Constrained and unconstrained vector fields. In order to exploit this decomposition algorithmically, we may think of the reaction coordinate $\dot{c}(q)=c^{\prime}$ as a holonomic constraint $T \Sigma$. We then observe that it is possible to write the force $f$ as a sum of the constraint force and a connection term both of which can be easily computed. This decomposition appeared as a result in $[1,2]$, yet the exact geometrical meaning of what was called "geometric correction" remained unclear. In fact it was pointed out in [9] that an alternative, more intrinsic definition of free energy which is used in the community of transition state theorists leads to the force $f$ as the Cartesian constraint force $\lambda D_{q} c$ but without the connection term.

If we carefully separate both vertical and horizontal velocity contributions, and then applying the vertical projection to the whole vector field $Z$, we obtain the force in the direction of the reaction coordinate

$$
f(q, \dot{q})=-\lambda\left(q, H_{q} \dot{q}\right)+\|n\|^{-4}\langle n, \dot{q}\rangle\langle n, \mathrm{~d} n[\dot{q}]\rangle, \quad q \in \Sigma,
$$

where $\lambda$ as defined in (A.4) is the constraint force, $n=D_{q} c$ is normal to $T_{q} \Sigma$, and $\mathrm{d} n[\dot{q}]$ denotes the directional derivative of the normal field along the vector $\dot{q}$. In some sense the connection 1-form can be understood as a constraint force that does work to the intrinsic motion on the constraint bundle $T \Sigma$, because it appears as a correction force in the way we have just explained.

We have to be a bit careful; the approach using holonomic constraints from the outset is very subtle as on the one hand we want to evaluate the force $f$ at $c=c^{\prime}$ regardless of $\dot{c}$, and on the other hand the holonomic constraint imposes the condition $\dot{c}(q)=0$ on the velocities. For that reason the supposedly attractive Routh construction $L^{\prime}=L-\lambda c$, or as well approaches using the Dirac bracket are bound to miss the point: either $\dot{c}=0$ on both sides of the equality, or $\dot{c} \neq 0$ in case of which $\lambda$ will not be the constraint force. In the end, the connection 1-form bridges this gap, collecting the velocities acting in the vertical direction.

1.3. Conditional forces from constrained averages. In principle the above expression for $f$ allows for an averaging scheme with respect to any distribution over the remaining coordinates. Mostly averaging is meant with regard to an 
invariant distributions of the system. Considering free energy calculations one uses the canonical distribution $\mu \propto \exp (-\beta H)$, which evaluated at $c=c^{\prime}$ is called the conditional density with the Hamiltonian $H(q, p)$ as sum of kinetic and potential energy. Intriguingly the constrained density $\mu_{\Sigma}$ is related to the conditional density in a simple way

$$
\mu_{\Sigma}(q, p)=\mu\left(q, H_{q}^{*} p\right), \quad q \in \Sigma,
$$

where $H_{q}^{*}=M H_{q} M^{-1}$ is the projection onto the horizontal (constrained) momentum subspace $T^{*} \Sigma$, and $M$ is the mass matrix. Thus $\mu_{\Sigma}$ is merely the full density $\mu$ restricted to $T^{*} \Sigma$. On the one hand this representation is very convenient because it does not rely on introducing local coordinates. On the other hand we catch a singularity in the Gaussian part of the canonical distribution, since the covariance matrix becomes rank-deficient. However this does not pose severe problems as we only want to compute expectation values from time series provided the flow is ergodic.

1.4. Conditional sampling with hybrid Monte-Carlo. From the considerations above it should be clear that we are interested in sampling the constrained distribution in position space only. It is known that the momentum contribution to the constraint force is related to the second fundamental form of the embedding, whereas the configuration part is due to the molecular potential only. The second fundamental form describes the curvature of the constraint surface in the surrounding space (extrinsic curvature). Taking the momentum average of the curvature term reveals that only the mean curvature of $\Sigma$ in $\mathbf{R}^{m}$ contributes to the force. A similar expression arises from averaging the connection 1-form. Thus with $\nu=n /\|n\|$ we get

$$
f(q)=-\|n\|^{-1}\left(\left\langle\nu, D_{q} U\right\rangle-\beta^{-1} \kappa-\beta^{-1} \omega[\nu]\right), \quad q \in \Sigma,
$$

where $\omega[\nu]=\|n\|^{-2}\langle n, \mathrm{~d} n[\nu]\rangle$ is the average connection 1-form. The first term is the vertical part of the molecular force field, $\beta$ is the inverse temperature parameter and $\kappa=\kappa(\sigma)$ denotes the mean curvature. The mean curvature stands for the metric entropy of the constraint surface, whereas the connection yields the average ambient space forcing on the surface.

Once the velocities have been averaged out, the conditional density in configuration space can be efficiently computed from the constrained canonical density using the blue moon sampling procedure [11]. In principle computing the mean force is not a big deal using any of the standard thermostat techniques. However they have to satisfy two major requirements: firstly the thermostat has to be consistent with constrained dynamics, and secondly the dynamics have to be ergodic with respect to to the constrained canonical density in the position variables.

It is well-known that the ordinary Nosé-Hoover thermostat suffers from ergodicity problems for certain classes of Hamiltonians $[12,13]$. This pathology can be removed by using extensions to the single-oscillator chain or the Andersen constant temperature thermostat $[14,15]$. But also there the sampling works well only if the dynamics are ergodic; and conditions to guarantee ergodicity are still lacking. Additionally all these more sophisticated methods have in common that due to their complexity they are relatively hard to implement, and that they require a careful adjustment of the involved parameters. Even worse, it is not clear a priori how these methods fit constrained symplectic integration; see [16] for a discussion on that topic. In particular for the Nosé-Hoover method the Lagrange multiplier $\lambda$ becomes dependent on the thermostat variables, which means that it can no longer be interpreted as the constraint force for the original subsystem. A promising alternative is to use stochastic 
high friction Langevin dynamics since it is ergodic for the systems under consideration. The procedure to generate the canonical ensemble on the constraint surfaces is described in [9]. However since our approach is heavily based on deterministic molecular motion we shall not discuss this method here.

We adopt the Hybrid Monte-Carlo (HMC) technique. Belonging to the class of Metropolis Monte-Carlo it is conceptually very simple, and it is perfectly designed to handle symplectic integration, as one can use standard integrators for constrained Hamiltonian systems. Moreover it is proven that the HMC dynamics are ergodic with respect to the positional density under rather weak conditions which are fulfilled for our purposes. We show that HMC generates indeed the constrained canonical density with an acceptance procedure that is similar to the usual Metropolis acceptance step. We further demonstrate how HMC can be efficiently used in connection with ordinary constrained molecular dynamics and show how the proposal can easily be generated. Note that the acceptance procedure also controls the numerical error, for the HMC rejects those moves that have too large energy fluctuations. We apply HMC to the problem of free energy calculations, but we emphasize that the algorithm can be used for any constrained MD runs.

\section{Some geometry}

2.1. Holonomic constraints. We start from the Lagrange function $L$ : $T \mathbf{R}^{m} \rightarrow \mathbf{R}$ for a molecule in an Euclidean configuration space that is defined by

$$
L(q, \dot{q})=\frac{1}{2}\langle M \dot{q}, \dot{q}\rangle-U(q),
$$

where $M$ is the diagonal mass matrix, and $U: \mathbf{R}^{m} \rightarrow \mathbf{R}$ is the molecular interaction potential. In order to keep a compact notation, we choose mass-scaled coordinates $q \mapsto M^{1 / 2} q$ which allows us to set $M=1$ in the following. By no means will this affect our considerations; all computations can be carried out with respect to the scaled coordinates using the standard inner product of $\mathbf{R}^{m}$.

Generally, a constraint for any Lagranian system is an affine subspace of the tangent space $T_{q} \mathbf{R}^{m}$ for each $q \in \mathbf{R}^{m}$. Let $c: \mathbf{R}^{m} \rightarrow \mathbf{R}$ be any smooth and regular vector-valued function. We define the configurational manifold of constraints as

$$
\Sigma=\left\{q \mid c(q)=c^{\prime}\right\} .
$$

Assume that the gradient $n=D_{q} c$ nowhere vanishes. Then $\Sigma$ is a proper submanifold of codimension one in $\mathbf{R}^{m}$, and the 1-form $\omega=n_{i} \mathrm{~d} q^{i}$ determines a distribution $\mathcal{D} \subset$ $T \mathbf{R}^{m}$ of dimension $m-1$ as the collection of

$$
\mathcal{D}(q)=\operatorname{ker} n(q) .
$$

Now recall the following notion: a constraint is holonomic if the distribution $\mathcal{D}$ is involutive, i.e., for any two vector fields $X, Y \in \mathcal{D}$ their Lie derivative $\nabla_{X} Y-\nabla_{Y} X$ lies in $\mathcal{D}$. Note that $\dot{q} \in \mathcal{D}$, or equivalently $\dot{c}(q)=0$ is sometimes referred to as the hidden constraint although it is the proper constraint.

The Frobenius Theorem asserts that $\mathcal{D}$ is involutive if and only if it is integrable $[17,18]$. This is equivalent to state that $\Sigma$ is an integral manifold of the vector field generated by $\mathcal{D}$. As a consequence we may introduce local coordinates $x^{1}, \ldots, x^{m-1}, y$ in a tubular neighbourhood of $\Sigma$, such that the constraint is expressed by

$$
y=0 \quad \text { and } \quad \dot{y}=0 .
$$


2.2. Lagrangian vector field. We shall shortly sketch the geometric framework that we need basically adopting the notation in [19]. Our aim is to study the vector field $Z$ which is generated by the Lagrangian (2.1) on the configurational submanifold (2.2). Consider a tubular neighbourhood of $\Sigma$, and define the normal bundle over $\Sigma$ by

$$
N \Sigma=\left\{(\sigma, \eta) \mid \sigma \in \Sigma, \eta \in N_{\sigma} \Sigma\right\} \subset \mathbf{R}^{m} \times \mathbf{R}^{m} .
$$

As there is a natural diffeomorphism of $N \Sigma$ into $\mathbf{R}^{m}$ given by $(\sigma, \eta) \mapsto \sigma+\eta$, we may express the Lagrangian (2.1) in a sufficiently small tubular neighbourhood of $\Sigma$ by

$$
L(\sigma, \eta, \dot{\sigma}, \dot{\eta})=\frac{1}{2}\langle\dot{\sigma}, \dot{\sigma}\rangle+\frac{1}{2}\langle\dot{\eta}, \dot{\eta}\rangle+U(\sigma+\eta) .
$$

The first step is to decompose the tangent spaces to $N \Sigma$ into horizontal and vertical parts, where vertical means the direction normal to $\Sigma$. For each $\sigma \in \Sigma$ consider a decomposition $T_{\sigma} \mathbf{R}^{m}=T_{\sigma} \Sigma \oplus N_{\sigma} \Sigma$ into tangent and normal space. Since we can naturally identify $T_{\sigma} \mathbf{R}^{m}$ with $\mathbf{R}^{m}$, we obtain a decomposition of $\mathbf{R}^{m}$. The corresponding orthogonal projections are defined by

$$
H_{\sigma}: T_{\sigma} \mathbf{R}^{m} \rightarrow T_{\sigma} \Sigma, \quad V_{\sigma}: T_{\sigma} \mathbf{R}^{m} \rightarrow N_{\sigma} \Sigma,
$$

such that the decomposition $X=H_{\sigma} X+V_{\sigma} X$ holds for an arbitrary tangent vector $X \in T_{\sigma} \mathbf{R}^{m}$. Now let $X, Y$ be any two vector fields on $\mathbf{R}^{m}$, and let $\nabla_{X} Y$ be the covariant derivative or affine connection in $\mathbf{R}^{m}$. Then the derivative $\nabla$ assigns another vector field $\nabla_{X} Y$ to $X, Y$ which we can decompose in the same way, namely

$$
\nabla_{X} Y=H_{\sigma} \nabla_{X} Y+V_{\sigma} \nabla_{X} Y
$$

In particular the Lagrangian vector field can be expressed in the language of covariant derivatives: take an arbitrary curve $q(t)$ in $\mathbf{R}^{m}$. Then the vector field

$$
Z(q, \dot{q})=\nabla_{\dot{q}} \dot{q}+\operatorname{grad} U
$$

is the second-order Lagrangian vector field on $T \mathbf{R}^{m}$, which boils down to the wellknown $\ddot{q}=-D_{q} U$ in case $q(t)$ is a solution of the Euler-Lagrange equations.

2.3. Coordinate expressions. Suppose $x=x(\sigma) \in \mathbf{R}^{m-1}$ provides a local coordinate map for $\Sigma$. Conversely, the inverse $\sigma=\sigma(x)$ is a local embedding of $\mathbf{R}^{m-1}$ onto $\Sigma \subset \mathbf{R}^{m}$. In accordance with the Frobenius Theorem, we introduce the local normal coordinate $y$ by $\eta(x, y)=y n(\sigma(x))$, where $y$ is an abbreviation for $c-c^{\prime}$ which is considered being an independent variable. Here $n(\sigma(x))$ denotes the non-unit normal to $\Sigma$, whereas the unit normal is denoted by $\nu(\sigma(x))$.

In the usual manner we identify all tangent spaces $T_{\sigma, \eta} N \Sigma$ with $m$-dimensional subspaces of $\mathbf{R}^{m} \times \mathbf{R}^{m}$. If $(\sigma(t), \eta(t))$ is a curve in $N \Sigma$ the tangent vectors are mapped into $\mathbf{R}^{m}$ due to $(\dot{\sigma}(0), \dot{\eta}(0)) \mapsto \dot{\sigma}(0)+\dot{\eta}(0)$. In accordance with the choice of local coordinates above the tangent vectors $\partial_{i} \in T_{\sigma, \eta} N \Sigma$ and $\partial_{y} \in T_{\sigma, \eta} N \Sigma$ correspond to the tangent vectors in $\mathbf{R}^{m} \times \mathbf{R}^{m 1}$

$$
v_{x}=\left(\partial_{i} \sigma(x) \dot{x}^{i}, y \mathrm{~d} n\left[\partial_{i} \sigma\right] \dot{x}^{i}\right), \quad v_{y}=(0, \dot{y} n(\sigma(x))) .
$$

\footnotetext{
${ }^{1}$ What follows is a rather serious abuse of notation as we will use the symbols $\partial_{i}$ and $\partial_{y}$ for partial derivatives as well as to denote the corresponding vectors in $T_{\sigma, \eta} N \Sigma$.
} 
Suppose $q(t)=\sigma(t)+\eta(t)$ is a curve in $N \Sigma$ and we want to know the component of $Z$ pointing in the direction of the coordinate $c$ at $c=c^{\prime}$. This is equal to asking for the normal component $\partial_{y}$ of the velocity vector $D_{q}$ at $y=0$. Namely

$$
D_{q}=\partial_{i} \sigma \partial^{i}+n \partial_{y}, \quad D_{q}=\left(\partial_{q^{1}}, \ldots, \partial_{q^{m}}\right)^{T} \in T_{\sigma} \mathbf{R}^{m}
$$

which clearly holds only on the surface $y=0$. Here $\partial_{i} \sigma$ and $n$ are vectors in $\mathbf{R}^{m}$. Note that we have raised one index to indicate summation over $i$. Hence we conclude

$$
\partial_{c}=\|n\|^{-1} V_{\sigma} D_{q}
$$

2.4. Force expressions. Using the previous arrangements we are ready to compute the force along $c$. We may address the molecular potential and the velocity field separately. Firstly note that due to (2.4) the contribution from the potential is related to the molecular force field $D_{q} U=\operatorname{grad} U$ in the following simple way

$$
\partial_{c} U=\|n\|^{-2}\left\langle n, D_{q} U\right\rangle \text {. }
$$

Omitting the potential in what follows we proceed with the calculation of the velocity field $v_{x}+v_{y}=\dot{\sigma}+\dot{\eta}$. From the bilinearity of the connection $\nabla$ we see that

$$
Z=\nabla_{\dot{\sigma}} \dot{\sigma}+\nabla_{\dot{\eta}} \dot{\sigma}+\nabla_{\dot{\sigma}+\dot{\eta}} \dot{\eta}
$$

where $\dot{\eta}=\dot{y} n$ on condition that $y=0$. Before carefully inspecting each single term, we may first of all note that $\nabla_{\dot{\eta}} \dot{\sigma}=\dot{y} \nabla_{n} \dot{\sigma}$. It is also true that $\nabla_{n} \dot{\sigma} \in T_{\sigma} \Sigma$, which follows from differenting the equation $\langle n, \dot{\sigma}\rangle=0$ along the normal direction, since

$$
\left\langle\nabla_{n} n, \dot{\sigma}\right\rangle=-\left\langle n, \nabla_{n} \dot{\sigma}\right\rangle
$$

where the left hand side of the equation is zero, because $\nabla_{n} n=\mathrm{d} n[n] \in N_{\sigma} \Sigma$. Therefore the vertical projection applied to $\nabla_{\dot{\eta}} \dot{\sigma}$ vanishes. Moreover space we can utilize a similar argument regarding the very first term $\nabla_{\dot{\sigma}} \dot{\sigma}$ to see that

$$
\left\langle n, \nabla_{\dot{\sigma}} \dot{\sigma}\right\rangle=-\left\langle\dot{\sigma}, \nabla_{\dot{\sigma}} n\right\rangle
$$

As both sides of the equation are different from zero we know that $\nabla_{\dot{\sigma}} \dot{\sigma}$ has a nonzero component in $N_{\sigma} \Sigma$. In fact the left hand side of the equation is known to be the second fundamental form. For the last term in (2.6) we obtain

$$
\nabla_{\dot{\sigma}+\dot{\eta}} \dot{\eta}=\dot{y} \nabla_{\dot{\sigma}+\dot{\eta}} n
$$

which means that the variations of the normal velocity $\dot{\eta}$ along a curve $(\sigma, \eta)$ are due to the variations of the moving normal frame $n(\sigma)$ and the independent variable $\dot{y}$. Due to (2.4) the interesting magnitude is the vertical component of $Z$

$$
V_{\sigma} Z=V_{\sigma}\left(\nabla_{\dot{\sigma}} \dot{\sigma}+\nabla_{\dot{\sigma}+\dot{\eta}} \dot{\eta}\right)
$$

Note that the expressions on the right hand side are quadratic forms in $\dot{z}=(\dot{x}, \dot{y})$, and observe the following fact: if we had started from the ordinary Euler-Lagrange equations, the right hand side of (2.7) would have the form $\Gamma_{j k}^{m} \dot{z}^{j} \dot{z}^{k}$, where the coefficients $\Gamma_{j k}^{m}$ denote the Christoffel symbols associated with the Levi-Civita connection on $T N \Sigma$, and $Z$ would be the geodesic spray of the pulled back Euclidean metric. 
2.5. Interpretation of force terms. We may split (2.7) and discuss the two parts separately. The first one is the extrinsic curvature of the hypersurface $\Sigma$ :

LEMMA 2.1. Let $\Sigma$ be a regularly immersed hypersurface, endowed with a Riemannian structure $\langle\cdot, \cdot\rangle_{x}=\langle\cdot, G(x) \cdot\rangle$. If $q(t)$ is a curve in $N \Sigma$, and if $\dot{q} \in T \mathbf{R}^{m}$ denotes its tangent vector field, then

$$
V_{\sigma} \nabla_{\dot{\sigma}} \dot{\sigma}=\langle S(q) \dot{q}, \dot{q}\rangle \nu
$$

whenever $q=\sigma$, where $\nu$ is the unit normal to $\Sigma$ and $S$ denotes the matrix of the Weingarten map $-\mathrm{d} \nu[\cdot]$ associated with the second fundamental form.

Proof. Let $\sigma(t) \in \Sigma$ be the projection of the curve $q(t)=(\sigma(t), \eta(t))$ onto the hypersurface. Differentiating $\langle\nu, \dot{\sigma}\rangle=0$ along the projected curve yields

$$
\left\langle\nu, \nabla_{\dot{\sigma}} \dot{\sigma}\right\rangle=-\left\langle\dot{\sigma}, \nabla_{\dot{\sigma}} \nu\right\rangle
$$

Note that since $\dot{\sigma}$ and $\nu$ are vectors in $\mathbf{R}^{m}$ the covariant derivative $\nabla_{\dot{\sigma}} \nu$ is the usual directional derivative $\mathrm{d} \nu[\dot{\sigma}]$, and we may write the right hand side as

$$
-\langle\dot{\sigma}, \mathrm{d} \nu[\dot{\sigma}]\rangle=-\left\langle\partial_{j} \sigma, \mathrm{d} \nu\left[\partial_{k} \sigma\right]\right\rangle \dot{x}^{j} \dot{x}^{k}
$$

The equation suggests to define a matrix $R(x) \in \mathbf{R}^{(m-1) \times(m-1)}$ which is the matrix of the Weingarten map $-\mathrm{d} \nu[\cdot]$ written in the local basis $\left\{\partial_{i} \sigma\right\}$ (see [24] or the Appendix for details). If $G(x) \in \mathbf{R}^{(m-1) \times(m-1)}$ denotes the metric tensor of $\Sigma$, then

$$
G_{j}^{h} R_{h k} \dot{x}^{j} \dot{x}^{k}=-\left\langle\partial_{j} \sigma, \mathrm{d} \nu\left[\partial_{k} \sigma\right]\right\rangle \dot{x}^{j} \dot{x}^{k}
$$

By carefully manipulating the vectors $\dot{x}$ we can extend the quadratic form to $\mathbf{R}^{m}$ : if we bear in mind that $\dot{\sigma}=D_{x} \sigma \dot{x}$, and if we set $J=D_{x} \sigma \in \mathbf{R}^{m \times(m-1)}$ then we find that $\dot{x}=G^{-1} J^{T} \dot{\sigma}$, for it is true that $G=J^{T} J$ is invertible.

As $\mathrm{d} \nu[\cdot]=\|n\|^{-1} H_{\sigma} \mathrm{d} n[\cdot]$ we may replace $\dot{\sigma} \in T_{\sigma} \Sigma$ by $\dot{q} \in T_{\sigma} \mathbf{R}^{m}$. Clearly $\dot{\sigma}$ is the horizontal part of an arbitrary tangent vector only on the hypersurface $\Sigma$, which means $\dot{\sigma}=H_{\sigma} \dot{q}$. But as $\dot{q}=\dot{\sigma}+\dot{\eta}$ with $\eta=y n(\sigma(x))$ the matrices $D_{x} \sigma$ and $D_{x} q$ coincide on the surface $y=0$, and we are allowed to perform

$$
\left\langle R G^{-1} J^{T} H_{\sigma} \dot{q}, J^{T} H_{\sigma} \dot{q}\right\rangle=\left\langle H_{\sigma} J R G^{-1} J^{T} \dot{q}, \dot{q}\right\rangle,
$$

where we have used the symmetry of $G^{-1}$ and $H_{\sigma}$ with respect to the product $\langle\cdot, \cdot\rangle$, and we have taken advantage of the idempotency of the horizontal projection. Hence it is reasonable to define a matrix $S(\sigma(x))$

$$
S_{j k} \dot{q}^{j} \dot{q}^{k}:=G_{j}^{h} R_{h k} \dot{x}^{j} \dot{x}^{k}, \quad \text { with } \quad S=H_{\sigma} J R G^{-1} J^{T} \in \mathbf{R}^{m \times m} .
$$

Apparently on $\Sigma$ the definition $S=H_{\sigma} J R G^{-1} J^{T}$ makes sense for vectors $\dot{q} \in \mathbf{R}^{m}$. If we take into account that $G^{-1} J^{T}$ is the pseudo inverse to $J$, we uncover $S$ as the Weingarten matrix in the standard Euclidean basis, i.e.,

$$
S(q)=-\|n(q)\|^{-1} H_{q} D_{q}^{2} c(q), \quad q \in \Sigma
$$

where $n(q)=D_{q} c(q)$ is the gradient of $c$ evaluated on the hypersurface.

REMARK 2.2. We have extended the smaller matrix $G R$ to the larger one $S$ in $\mathbf{R}^{m}$ without any ambiguities. The intriguing point however is: up to sign the second 
fundamental form is known to be the velocity contribution to the constraint force [25]. Hence we can uniquely assign it to the constraint force, although we have $\dot{y} \neq 0$ in the equations for the total vector field $Z$ (see also the Appendix).

The second term in (2.7) describes the coupling between the intrisic (constrained) flow and the normal vector field. Hence we address the connection part:

Lemma 2.3. Let all assumptions from the previous Lemma hold, and let $\mathrm{d} n[\cdot]$ denote the directional derivative of the normal field along some vector. Then

$$
V_{\sigma} \nabla_{\dot{\sigma}+\dot{\eta}} \dot{\eta}=\omega[\dot{q}] V_{\sigma} \dot{q}
$$

where $\omega[\cdot]=\|n\|^{-2}\langle n, \mathrm{~d} n[\cdot]\rangle$ is the connection 1-form of the normal frame $n$.

Proof. Set $\dot{q}=\dot{\sigma}+\dot{\eta}$, and note that we can replace the covariant derivative by the directional derivative $\mathrm{d} \dot{\eta}[\dot{q}]$. Resolving $\dot{\eta}=\dot{y} n$, where the independent variable $\dot{y}$ is not affected by the derivative, we find that

$$
V_{\sigma} \nabla_{\dot{\sigma}+\dot{\eta}} \dot{\eta}=\dot{y}\langle\nu, \mathrm{d} n[\dot{q}]\rangle \nu .
$$

Then exploiting that $\dot{y}=\|n\|^{-1}\langle\nu, \dot{q}\rangle$ on condition that $y=0$, and plugging this relation into the last equation we obtain the result with $\omega[\dot{q}]=\|n\|^{-2}\langle n, \mathrm{~d} n[\dot{q}]\rangle$.

REMARK 2.4. The connection 1-form $\omega[\cdot]$ is a vertical valued 1-form, which is associated with the connection $\|n\|^{-1} \mathrm{~d} n[\cdot]$ of the normal frame $n$. Unlike in case of a normalised basis $\nu$ for the normal bundle to a hypersurface the connection 1-form does not vanish. In the latter case $\|\nu(\sigma)\|^{2}=1$ is conserved by the flow on $\Sigma$ and therefore $\langle\nu, \mathrm{d} \nu[\cdot]\rangle=0$. Contrarily in our case the normal motion is coupled to the motion on the surface, which is responsible for our specific choice of a normal frame.

Let us summarise the last few steps: after projecting the force field onto the vertical direction according to (2.4) and taking the norm we get from the equations $(2.5),(2.8)$ and $(2.9)$ the final expression for the force along the reaction coordinate

$$
\left.f(q, \dot{q})=-\lambda\left(q, H_{q} \dot{q}\right)\right)+\|n\|^{-2}\langle n, \dot{q}\rangle \omega[\dot{q}],
$$

which holds upon $q \in \Sigma$, and in correspondence with (A.4),

$$
\lambda\left(q, H_{q} \dot{q}\right)=-\|n\|^{-2}\left\langle n, D_{q} U\right\rangle+\|n\|^{-1}\langle S(q) \dot{q}, \dot{q}\rangle,
$$

is the constraint force with respect to the coordinate $c$.

\section{Free energy as potential of mean force}

In accordance with the MD literature we shall call the parameter $c^{\prime} \in \mathbf{R}$ the reaction coordinate. It determines the hypersurface $\Sigma=c^{-1}\left(c^{\prime}\right)$ over which we will have to average the force. Without closer specification at this stage we let $\mathbf{E}\left[\cdot \mid c=c^{\prime}\right]$ denote the average over the hypersurface $\Sigma$ (conditional average). If $f(q, \dot{q})$ is the force acting along $c$ on condition that $q \in \Sigma$, then the mean force reads

$$
f_{\text {mean }}\left(c^{\prime}\right)=\mathbf{E}\left[f(q, \dot{q}) \mid c=c^{\prime}\right] .
$$

In case the average is understood with respect to the canonical density $\mu \propto \exp (-\beta H)$ it is easy to show [26] that the potential which corresponds to the mean force is the thermodynamical Helmholtz free energy

$$
\begin{aligned}
F\left(c^{\prime}\right) & =-\beta^{-1} \ln \int \exp (-\beta H(q, p)) \delta\left(c(q)-c^{\prime}\right) \mathrm{d} q^{1} \wedge \ldots \wedge \mathrm{d} p^{m} \\
& =-\beta^{-1} \ln \int_{q \in \Sigma} \exp (-\beta H(q, p))\left\|D_{q} c(q)\right\|^{-1} \mathrm{~d} \sigma(q),
\end{aligned}
$$


where $H=\langle\dot{q}, p\rangle-L$ denotes the molecular Hamiltonian, $\beta$ is the inverse temperature, and $\mathrm{d} \sigma$ is the ordinary surface element (see the Appendix). In principle the last equation defines the free energy, and the mean force identity above pops out after taking the partial derivative with respect to the parameter $c^{\prime}$.

REMARK 3.1. As an algorithmic approach it is common to compute the free energy by integrating the mean force, a method which is known as thermodynamic integration [6]. In our case we want to use constrained simulations in order to compute the mean force. After having derived the expression for the force $f$ one point remains to be clarified: how can the conditional expectation be obtained from constrained simulations? We will address this questions in the following subsections.

3.1. Remark on the canonical density I. We are aiming at computing the mean force in a molecular ensemble at constant temperature. This means that we have to average over the canonical ensemble which we shall denote by $\mu$. In case that $q$ is restricted to lie on the hypersurface $\Sigma$ we may call $\mu(q, p)$ with $q \in \Sigma$ the conditional density. Since this makes no difference on the conceptual level, we stick to the symbol $\mu$ in either case. In order to state what the canonical density is we introduce the molecular Hamiltonian $H: T^{*} \mathbf{R}^{m} \rightarrow \mathbf{R}$ by

$$
H(q, p)=\langle p, \dot{q}\rangle-L(q, \dot{q}) \quad \text { with } \quad p_{i}=\frac{\partial L}{\partial \dot{q}^{i}} \in T^{*} \mathbf{R}^{m}
$$

The canonical invariant measure of the dynamics generated by $H$ then has the density

$$
\mu(q, p)=Z^{-1} \exp (-\beta H(q, p)), \quad \text { with } Z=\int \exp (-\beta H(q, p)) \Lambda
$$

using the common notation $\beta=1 / T$ for the inverse temperature with the Boltzman constant set to unity. Here, $\Lambda=\mathrm{d} q^{1} \wedge \ldots \wedge \mathrm{d} p^{m}$ is the standard volume Liouville volume form. As well as any other function $\chi(H)$ of the Hamiltonian $\mu$ is a stationary solution of the Liouville equation $\dot{\chi}=\{\chi, H\}$ for $\{\chi, H\}: T^{*} \mathbf{R}^{m} \rightarrow \mathbf{R}$ denoting the Poisson bracket on $T^{*} \mathbf{R}^{m}$. We can compute the momentum average of (2.10):

Proposition 3.2. Let $\Sigma=c^{-1}\left(c^{\prime}\right)$ be the configurational manifold of constraints with mean curvature $\kappa$ in $\mathbf{R}^{m}$, and let $\nu=n /\|n\|$ be the unit normal to $T \Sigma$. Suppose $q \in \Sigma$. If we average out the velocities in (2.10) with respect to to the canonical density the force as a function in configuration space is given by

$$
f(q)=-\|n\|^{-1}\left(\left\langle\nu, D_{q} U\right\rangle-\beta^{-1} \kappa-\beta^{-1} \omega[\nu]\right),
$$

where $\omega[\nu]=\|n\|^{-2}\langle n, \mathrm{~d} n[\nu]\rangle$ is the averaged connection 1-form of the normal frame.

Proof. It suffices to consider the velocity contributions only. Setting $\dot{q}=p$ in (2.10) which we are allowed to do due to $M=\mathbf{1}$, we consider integrals of the type

$$
\int\langle K(q) p, p\rangle \exp (-\beta / 2\langle p, p\rangle) \mathrm{d} p^{1} \wedge \ldots \wedge \mathrm{d} p^{m}
$$

The task is to compute the covariance matrix of the Gaussian density. Due to the shape of the Gaussian density only the diagonal terms $p^{i} p_{i}$ survive, i.e.,

$$
\begin{aligned}
& \int\langle K(q) p, p\rangle \exp (-\beta / 2\langle p, p\rangle) \mathrm{d} p^{1} \wedge \ldots \wedge \mathrm{d} p^{m} \\
= & \beta^{-1} \operatorname{tr}(K(q)) \int \exp (-\beta / 2\langle p, p\rangle) \mathrm{d} p^{1} \wedge \ldots \wedge \mathrm{d} p^{m} .
\end{aligned}
$$


It is well-known that in case $K(q)=S(q)$, the matrix of the Weingarten map, the trace $\operatorname{tr}(S)$ is the expression for the mean curvature $\kappa$ of $\Sigma$ in $\mathbf{R}^{m}$ up to a normalisation factor $1 / n$. Concerning the connection term we firstly observe that only the vertical momenta $\langle\nu, p\rangle \neq 0$ contribute on average. Taking the trace over the vertical subspace we obtain $\operatorname{tr}(K)=\langle n, \mathrm{~d} n[\nu]\rangle$ and thus the result.

REMARK 3.3. Carefully inspecting the last equation a manifest physical interpretation for the curvature contribution reveals: recall the thermodynamical definition of the Helmholtz free energy $F=U-T S$, where $T=1 / \beta$. The first part $U$ is the energetic contribution which is equivalent to our potential function $U$ in the equations of motion. Thus comparing $F$ to the formula (3.2) for the average force we claim that the mean curvature represents the entropic part of the free energy. The averaged connection is again a connection 1-form [27], and can be considered as the average ambient space force arising from the normal velocities. Note our result coincides with the previously derived results in [1, 8, 2].

3.2. Remark on the canonical density II. In the same manner as we have introduces the canonical density we can construct the constrained canonical density $\mu_{\Sigma}$. As we can express the constrained density in terms of the intrinsic Hamiltonian $H_{\Sigma}\left(x, p_{x}\right)=\left\langle\dot{x}, p_{x}\right\rangle-L\left(x, \dot{x}, c^{\prime}, 0\right)$, where $p_{x}$ denotes the conjugate momentum to $x$, we may express $\mu_{\Sigma}$ using local coordinates. That is

$$
\mu_{\Sigma}\left(x, p_{x}\right)=Z_{\Sigma}^{-1} \exp \left(-\beta H_{\Sigma}\left(x, p_{x}\right)\right), \quad \text { with } Z_{\Sigma}=\int \exp \left(-\beta H_{\Sigma}\left(x, p_{x}\right)\right) \Lambda_{\Sigma}
$$

with $\Lambda_{\Sigma}=\mathrm{d} x^{1} \wedge \ldots \wedge \mathrm{d} p_{x}^{m-1}$. However we might be willing to stick to the original coordinates and state the relation between $\mu$ and $\mu_{\Sigma}$ in terms of $q$ and $p$ rather than $x$ and $p_{x}$. To this end we define a family of vector fields $X_{\lambda}$, which are generated by the family of Hamiltonians $H_{\lambda}=H+\lambda\left(c(q)-c^{\prime}\right)$. The parameter $\lambda$ can be chosen in such a way, that the function $c(q)$ is a first integral of $X_{\lambda}$, and that the corresponding vector field is again of Hamiltonian type on the restricted phase space $T^{*} \Sigma$. A procedure which establishes a relation between vector fields generated by $H$ and $H_{\lambda}$ is due to Dirac [28]. Taking the Poisson bracket of $z=(q, p)$ with the parametrised Hamiltonian $H_{\lambda}$ on condition that $c=c^{\prime}$ and $\dot{c}=0$ we obtain [29]

$$
X_{\lambda}=X_{H}+\{X, c\}\{c, \dot{c}\}^{-1}\{\dot{c}, H\}=X_{\lambda}+\lambda D_{q} c .
$$

The last equation states that the constrained vector field is the molecular vector field $X_{H}=\{z, H\}$ plus the constraint force $\lambda D_{q} c$ both evaluated at $c=c^{\prime}$ and $\dot{c}=0$. The last identification requires that we have fixed the parameter as

$$
\lambda=\{c, \dot{c}\}^{-1}\{\dot{c}, H\}
$$

which is thus no longer an independent parameter but rather a function of $q$ and $p$. The condition that $\{c, \dot{c}\}$ be invertible is called the cosymplecticity condition on $\Sigma$, which is sufficient for the constrained dynamics being Hamiltonian [30]. It guarantees that the symplectic form on $\Sigma$ is non-degenerate and closed, which obviously cannot be taken for granted without imposing $\dot{c}=0$. At the same time, it is a necessary condition to ensure that the bracket on the left hand side is well-defined. It is frequently stated, that the constrained dynamics were not Hamiltonian [31, 32]. This is wrong as far as the dynamics on the constraint surface are concerned, for the above principle states that the resctriction of the full Hamiltonian vector field to the constraint surface is 
the same as the vector field that is generated by the restricted Hamiltonian. The latter one is clearly a Hamiltonian vector field.

We want to use this relation to compute the constrained density from the canonical density $\mu=Z^{-1} \exp (-\beta H)$. We endeavour the relation (3.3) and find for $p \in T_{\sigma}^{*} \Sigma$

$$
\dot{\mu}_{\Sigma}(q, p)=\dot{\mu}(q, p)+\{\mu(q, p), c\}\{c, \dot{c}\}^{-1}\{\dot{c}, H\}=\dot{\mu}(q, p) .
$$

This identity is owed to the fact, that $\{\mu, c\}$ is proportional to $\langle p, n(\sigma)\rangle$, which is zero on condition the constraint is satisfied. Note, that since we are using mass-scaled coordinates, we can identify coordinates on $T^{*} \mathbf{R}^{m}$ with those on $T \mathbf{R}^{m}$ and therefore $n=D_{q} c$ is perpendicular to $p$, too. This is a handy result, because it shows that the unconstrained canonical density $\mu$ evaluated on $T^{*} \Sigma$ is indeed the constrained invariant density $\mu_{\Sigma}$ in Euclidean coordinates, or that

$$
\mu_{\Sigma}(q, p)=\mu\left(q, H_{q}^{*} p\right), \quad q \in \Sigma,
$$

where $H_{q}^{*}: T_{q}^{*} \mathbf{R}^{m} \rightarrow T_{q}^{*} \Sigma$ is equal to $H_{q}$ due to the mass scaling assumption.

3.3. Conditional and constrained averages. So far we have put away any questions concerning the computational aspects of the conditional measure: how can the conditional measure be obtained from the constrained dynamics? It is a straight consequence of its definition that the constrained Liouville form is the restricted standard Liouville form $\Lambda \mapsto \Lambda_{\Sigma}$ (see Remark 4.3 below). In contrast, the volume form corresponding to a conditional measure cannot be a Liouville form, because it is odd dimensional; even worse, it cannot be invariant with respect to the Hamiltonian flow.

Though we will not pick up this topic in too much detail, the basic problem is easy to understand: according to the cosymplecticity condition the canonical choice to $\mathrm{d} c=0$ would be $\mathrm{d} p_{c}=0$. If the vertical momenta are left free, $\mathrm{d} p_{c} \neq 0$ creates a nontrivial functional determinant occuring in the volume form of the conditional average which indeed equals the blue moon factor [31, 11].

Proposition 3.4. Let $\mu(q, p)$ denote the conditional probability density and assume that it is separable, i.e., $\mu(q, p)=Q(q) P(p)$ for each $q \in \Sigma$. Likewise we let $\mu_{\Sigma}(q, p)$ denote the constrained density. Then with $n(q)=D_{q} c(q)$ the following identity holds

$$
\int \mathcal{O}(q) \mu \mathrm{d} q^{1} \wedge \ldots \wedge \mathrm{d} p^{m}=\int \mathcal{O}(q)\|n(q)\|^{-1} \mu_{\Sigma} \mathrm{d} q^{1} \wedge \ldots \wedge \mathrm{d} p^{m}
$$

for any integrable function $\mathcal{O}(q)$ and $q \in \Sigma$.

Proof. The result follows from the transformation properties of integrals. Recall that mass scaling $q \mapsto M^{1 / 2}, p \mapsto M^{-1 / 2} p$ leaves the symplectic form invariant and so the Liouville measure remains unrendered. Moreover we can identify coordinates in $T \mathbf{R}^{m}$ with those in $T^{*} \mathbf{R}^{m}$ such that the constraint equation $\dot{c}(\sigma)=0$ reads

$$
\langle n(\sigma), \dot{q}\rangle=\langle n(\sigma), p\rangle=0, \quad \sigma \in \Sigma
$$

defining an integrable codistribution $\mathcal{D}^{*}(\sigma) \subset T_{\sigma}^{*} \mathbf{R}^{m}$, where $T_{\sigma}^{*} \mathbf{R}^{m}$ will be naturally identified with $\mathbf{R}^{m}$ in what follows. Suppose $n_{m} \neq 0$. Then we can locally parameterise the constrained momenta by a map $\xi(u)$

$$
\xi\left(u^{1}, \ldots, u^{m-1}\right)=\left(u^{1}, \ldots, u^{m-1},-n_{m}^{-1} n^{j} u_{j}\right) \in \mathbf{R}^{m}
$$

with $p_{j}=u_{j}$, and $j$ is running from 1 to $m-1$. Note that the $n_{j}, n_{m}$ are functions of $\sigma$ only. We shall omit $\mathcal{O}(q)=\mathcal{O}(\sigma+\eta)$ since it is not affected by the momentum 
integration, and compute the constrained momentum average simply as the surface integral of the conditional density over the codistribution $\mathcal{D}^{*}(\sigma)$, i.e.

$$
\int_{\mathbf{R}^{m} \cap \mathcal{D}^{*}} P(\mathrm{~d} p)=\int_{\mathbf{R}^{m-1}}(P \circ \xi)(u) \operatorname{vol}\left(D_{u} \xi\right) \mathrm{d} u_{1} \wedge \ldots \wedge \mathrm{d} u_{m-1}
$$

The matrix volume occurring in the last equation is the analogue of the functional determinant for the map $\xi$. Endeavouring the Theorem of Cauchy-Binet we find [33]

$$
\operatorname{vol}\left(D_{u} \xi\right)=\sqrt{\operatorname{det}\left(D_{u} \xi^{T} D_{u} \xi\right)}=\sqrt{1+n_{m}^{-2} n^{j} n_{j}}
$$

Note that $\xi(u)$ is an embedding; contrarily $u(\xi)$ is a local coordinate map for $\mathcal{D}^{*}(\sigma)$. On the other hand we can write down the conditional density changing variables in the spirit of the previous sections: $\operatorname{set} \dot{c}(\sigma)=\gamma$, and define a family of maps

$$
p_{\gamma}^{m}\left(p^{1}, \ldots, p^{m-1}\right)=n_{m}^{-1}\left(\gamma-n^{j} p_{j}\right),
$$

where the free parameter $\gamma$ acts like a coordinate. We may change coordinates according to $\zeta\left(p^{1}, \ldots, p^{m}\right)=\left(p^{1}, \ldots, p^{m-1}, \gamma\right)$. As before we slightly abuse notation, and denote the map as well as the new coordinates by $\zeta$. Now the corresponding functional determinant reduces to $\operatorname{det}\left(D_{\zeta} p\right)=\partial_{\gamma} p^{m}$. Therefore

$$
\int_{\mathbf{R}^{m}} P(\mathrm{~d} p)=\int_{\mathbf{R}^{m}}\left(P \circ \zeta^{-1}\right)(p, \gamma)\left|n_{m}\right|^{-1} \mathrm{~d} p^{1} \wedge \ldots \wedge \mathrm{d} \gamma
$$

follows from pulling back the integrand by $\zeta$. In fact the surface integral in (3.6) corresponds to the constrained density $P_{\Sigma}$ for the momenta. Provided that both $\mu$ and $\mu_{\Sigma}$ are normalised we can combine the equations (3.6) and (3.7) to conclude that

$$
\int P_{\Sigma}(\sigma, \mathrm{d} p)\left(\int P(\mathrm{~d} p)\right)^{-1}=\sqrt{n_{m}^{2}+n^{j} n_{j}}=\|n(\sigma)\| .
$$

Hence the result follows from the requirement that $\mu$ and $\mu_{\Sigma}$ are normalised.

REMARK 3.5. This Proposition is a rather general version of the famous Fixman Theorem, which is the basis for the classical blue moon ensemble method [11, 34]. To the best of the author's knowledge the sole way in the MD literature to prove "blue moon" in the context of Hamiltonian dynamics relies on the specific form of the Gaussian-like momentum distribution of the canonical density. In this case the weight arises from the ratio of the determinants of the covariance matrices of constrained and conditional densities, respectively [26]. However the considerations above show that the weight is a merely geometric property, and that it is independent of the associated density provided only that it is separable. In principle the assumption that the observable $\mathcal{O}$ is a purely configurational function only may be weakened in the sense that it must not depend on the vertical momenta.

\section{Hybrid Monte-Carlo}

So far however, it is by no means obvious how a discretisation of (A.2) should put forth a canonical measure rather than the microcanonical one. In order to calculate canonical averages by means of (3.2), we want to develop an algorithmic concept that allows for computing canonical averages, and that does not involve any artificially added heat bath dynamics. 
We start with a local representation according to Section $\S 2$; the invariant measure of the constrained dynamics is given by (3.1). On $T^{*} \Sigma$ we cannot separate momentum dependent parts from position dependent parts, and so we write $\mu_{\Sigma}=Q(x) P_{x}(u)$, indicating that the kinetic energy depends on the position coordinates. In order to see how the constrained HMC scheme works with $\mu_{\Sigma}$, we let the one-parameter semigroup $\varphi^{\tau}$ denote a symmetric and symplectic flow map that is consistent with the constrained equations of motion. Then for a single integration step $\left(x^{\prime}, u^{\prime}\right)=\varphi^{\tau}(x, u)$ the HMC acceptance function is defined by

$$
a\left(x, x^{\prime}\right)=\min \left\{1, \frac{\mu_{\Sigma}\left(x^{\prime},-u^{\prime}\right) \Lambda^{\prime}}{\mu_{\Sigma}(x, u) \Lambda}\right\}=\min \left\{1, \frac{Q\left(x^{\prime}\right) P_{x^{\prime}}\left(u^{\prime}\right)}{Q(x) P_{x}(u)}\right\} .
$$

This definition relies on the symmetry of $P_{x}(u)$ and the Liouville form $\Lambda$ with respect to momentum inversion $u \mapsto-u$ as well as on the invariance of $\Lambda$ under $\varphi^{\tau}$, since the flow map is symplectic (see the Remark below). We have to show that propagating according to the HMC transition function - which is the acceptance function $a\left(x, x^{\prime}\right)$ for a deterministic flow - leaves the canonical density $\mu_{\Sigma}$ invariant. This is by no means trivially fulfilled, since our Hamiltonian system has no unique invariant measure. The following statement holds

Proposition 4.1. Set the accepted proposal to $x^{*}=\left(\pi \circ a \circ \varphi^{\tau}\right)(x, u)$ with an initial momentum $u$ distributed according to $\mu_{\Sigma}(x, \cdot)=Q(x) P_{x}(\cdot)$. Furthermore let $\vartheta\left(x^{*}, u^{\prime}\right)$ denote the distribution of the position coordinates after one HMC step. Then for any position dependent observable $\mathcal{O} \in L^{1}\left(\mu_{\Sigma}\right)$ the identity $\mathbf{E}_{\vartheta}[\mathcal{O}]=\mathbf{E}_{Q}\left[m\left(x^{*}\right) \mathcal{O}\right]$ holds, where $m\left(x^{*}\right)$ depends only on the Riemannian structure of the constraint surface $\Sigma$.

Proof. For notational convenience, we drop the subscript $\Sigma$. Suppose the initial position $x$ follows the canonical distribution $\mu(\cdot, u)$. Then for a given $x$ we draw a momentum vector from $P_{x}(u)$, and propagate a single time-step according to $\varphi^{\tau}$. Following [35], this yields for the expectation value

$$
\begin{aligned}
\mathbf{E}_{\vartheta}[\mathcal{O}]=\int & \mathcal{O}\left(\mu\left(\varphi^{-\tau}\left(x^{*}, u^{\prime}\right)\right) \min \left\{1, \frac{\mu\left(x^{*}, u^{\prime}\right)}{\mu\left(\varphi^{-\tau}\left(x^{*}, u^{\prime}\right)\right)}\right\} \operatorname{det}\left(\mathbf{D} \varphi^{-\tau}\right)\right. \\
& \left.+\mu\left(x^{*}, u^{\prime}\right)\left(1-\min \left\{1, \frac{\mu\left(\varphi^{-\tau}\left(x^{*}, u^{\prime}\right)\right)}{\mu\left(x^{*}, u^{\prime}\right)}\right\}\right)\right) \Lambda^{\prime}
\end{aligned}
$$

where the first term of the right-hand side of the equation comes from the acceptance, the second one stems from the rejection step. Furthermore we made use of the symmetry of $\varphi^{\tau}$ in the second term. On condition that $\varphi^{\tau}$ is $\operatorname{symplectic}, \operatorname{det}\left(\mathbf{D} \varphi^{-\tau}\right)$ equals one. From integrating out the momenta we easily find that

$$
\begin{aligned}
\mathbf{E}_{\vartheta}[\mathcal{O}] & =\int \mathcal{O}\left(\mu\left(x^{*}, u^{\prime}\right)+\min \left\{\mu\left(\varphi^{-\tau}\left(x^{*}, u^{\prime}\right)\right), \mu\left(x^{*}, u^{\prime}\right)\right\}\right. \\
& \left.-\min \left\{\mu\left(x^{*}, u^{\prime}\right), \mu\left(\varphi^{-\tau}\left(x^{*}, u^{\prime}\right)\right)\right\}\right) \Lambda^{\prime} \\
& =\int \mathcal{O} \mu\left(x^{*}, u^{\prime}\right) \Lambda^{\prime}=\int \mathcal{O} \sqrt{\operatorname{det} G\left(x^{*}\right)} Q\left(x^{*}\right) \mathrm{d} x^{* 1} \wedge \ldots \wedge \mathrm{d} x^{* m-n}
\end{aligned}
$$

The square root in the very last expression originates from the inverse metric tensor $G^{-1}$ on the horizontal subbundle $T^{*} \Sigma$. We therefore have $m=\sqrt{\operatorname{det} G}$ which is the correct marginal distribution in the position variables. 
COROLlary 4.2. Let $O(q)$ with $q \in \Sigma$ stand for the restricted positional density in Cartesian coordinates and let $\vartheta(x)$ denote the density after one HMC step. Then $\mathbf{E}_{\vartheta}[\tilde{\mathcal{O}}(x)]=\mathbf{E}_{Q}[\mathcal{O}(q)]$ with $\tilde{\mathcal{O}}(x)=\mathcal{O}(q)$ holds true.

Proof. Recall that $\mathrm{d} \sigma=\operatorname{vol}(J) \mathrm{d} x$, where $J=D_{x} \sigma$ is the Jacobian of the parameterisation $\sigma(x)$ of $\Sigma$. Also note that $G=J^{T} J$ is the corresponding metric tensor, i.e., $\operatorname{vol}(J)=\sqrt{\operatorname{det} G}$. Using the transformation rule for integrating over a form yields

$$
\begin{aligned}
\mathbf{E}_{\vartheta}[\tilde{\mathcal{O}}(x)] & =\int \tilde{\mathcal{O}}(x) Q(x) \sqrt{\operatorname{det} G(x)} \mathrm{d} x^{1} \wedge \ldots \wedge \mathrm{d} x^{m-n} \\
& =\int \mathcal{O}(\sigma) Q(\sigma) \mathrm{d} \sigma^{1} \wedge \ldots \wedge \mathrm{d} \sigma^{m} \\
& =\int \mathcal{O}(q) Q(q) \mathrm{d} q^{1} \wedge \ldots \wedge \mathrm{d} q^{m}=\mathbf{E}_{Q}[\mathcal{O}]
\end{aligned}
$$

where the last step is justified since the range of $D_{x} q$ is equal to the range of $D_{x} \sigma$ if the derivatives are evaluated on $\Sigma$, and we can replace $\sigma$ by $q \in \Sigma \cap \mathbf{R}^{m}$.

So, why does HMC give a symplectic and time-reversible mapping, too? Certainly, the HMC inherits symplecticity from the discrete flow $\varphi^{\tau}$. Time-reversibility can be verified by checking detailed balance:

$$
\mu(x, u) a\left(x, x^{\prime}\right)=\mu(x, u) \min \left\{1, \frac{\mu\left(x^{\prime}, u^{\prime}\right)}{\mu(x, u)}\right\}=\min \left\{\mu\left(x^{\prime}, u^{\prime}\right), \mu(x, u)\right\} .
$$

The last equation is symmetric in regard to the primed and unprimed variables. Hence the detailed balance condition is satisfied, for $\mu(x, u) a\left(x, x^{\prime}\right)=\mu\left(x^{\prime}, u^{\prime}\right) a\left(x^{\prime}, x\right)$.

REMARK 4.3. The invariance of the Liouville form is a consequence of the symplecticity of the flow map $\varphi$. Bear in mind that the Liouville form is related to the symplectic form $\Omega$ by the coordinate-free definition [25]

$$
\Lambda=\frac{(-1)^{k(k-1) / 2}}{n !} \Omega \wedge \ldots \wedge \Omega,
$$

where the right hand side contains an $n$-fold exterior product. As a fundamental property the pull-back of an exterior product by a map is the exterior product of the pull-back, i.e., $(\alpha \wedge \beta) \circ \varphi^{-1}=\left(\alpha \circ \varphi^{-1}\right) \wedge\left(\beta \circ \varphi^{-1}\right)$. Hence we can conclude from symplecticity of the flow map, i.e., $\Omega \circ \varphi^{-1}=\Omega$ that $\Lambda^{\prime}=\Lambda$ above.

\section{Appendix A. More geometry.}

A.1. Fixing local coordinates. Let us now address the practical issues of finding local coordinates. A hypersurface $\Sigma$ defined by the scalar equation $c(q)=0$ is called non-singular, if $D_{q} c \neq 0$ on the surface [36, 37]. Then, the following Lemma holds:

Lemma A.1. Be $q^{*} \in \Sigma$ any non-singular point of the hypersurface, and let $U_{\epsilon}\left(q^{*}\right)$ denote a sufficiently small tubular neighbourhood including that point. Then, there is a parameterisation of $\Sigma$ in $U_{\epsilon}\left(q^{*}\right)$ given by $\left\{q^{1}, \ldots, q^{m-1}, h\right\}$, where $h: \mathbf{R}^{m-1} \rightarrow \mathbf{R}$ is the local inverse of $c$ defined by $q^{m}=h\left(q^{1}, \ldots, q^{m-1}\right)$.

Proof. Suppose, $q^{*}$ is a non-singular point of $\Sigma$, and say $\partial_{m} c \neq 0$ at $q^{*} \in \Sigma$. In this case, the Implicit Function Theorem guarantees that we can locally solve the equation $c(q)=0$ for $q^{m}$, obtaining a smooth function of the remaining coordinates. Let this 
function be denoted by $q^{m}=h\left(q^{1}, \ldots, q^{m-1}\right)$, such that $c\left(q^{1}, \ldots, q^{m-1}, h\right)=0$. Then the set $\left\{\sigma^{i}\right\}=\left\{q^{1}, \ldots, q^{m-1}, h\left(q^{1}, \ldots, q^{m-1}\right)\right\}$ is a parameterisation in $\Sigma \cap U_{\epsilon}\left(q^{*}\right)$.

Note, that the assumption $\partial_{m} c \neq 0$ does not affect our considerations, for we can always choose a different parameterisation $\tilde{\sigma}$, say with $q^{1}=\tilde{h}\left(q^{2}, \ldots, q^{m}\right)$. Then, if $c$ is at least of class $C^{2}$, the transition functions $\psi=\sigma \circ \tilde{\sigma}^{-1}$ are $C^{2}$, too. Thus $\Sigma$ will be globally smooth. Furthermore, if we bear in mind the identity $q^{m}=h$, we obtain from implicit differentiation of $c\left(q^{1}, \ldots, q^{m-1}, h\right)=0$

$$
\partial_{i} h=-\partial_{i} c\left(\partial_{m} c\right)^{-1}, \quad q \in U_{\epsilon}\left(q^{*}\right) .
$$

Now, defining local coordinates for the normal bundle $N \Sigma$ is pretty much straightforward: take local coordinates $\left\{x^{1}, \ldots, x^{m-1}, c\right\}$ with $x^{i}=q^{i}$ for $i=1, \ldots, m-1$. Then, $\left\{x^{1}, \ldots, x^{m-1}\right\}$ provide local coordinates on $\Sigma$ and $\sigma=\sigma(x)$ is an embedding of $\Sigma$ into $\mathbf{R}^{m}$. We may regard $y \in \mathbf{R}$ as normal coordinates for $N_{\sigma} \Sigma=\operatorname{span}\{n(\sigma)\}$, such that points on $\Sigma$ are fixed by $y=c-c^{\prime}=0$, where $n(\sigma)$ stands normal to $\Sigma$.

A.2. Geometry of the constraint force. We like to get physical or geometrical insight into the different contributions to the constraint force. Rather that using local coordinates we may apply some sort of index reduction technique for differential algebraic equations. Utilising the Theorem on Lagrange multipliers the constrained Euler-Lagrange equations are [25]

$$
\frac{d}{d t} D_{\dot{q}} L(q, \dot{q})=D_{q} L(q, \dot{q})-\lambda D_{q} c(q), \quad c(q)=0 .
$$

which leads to the standard Newtonian equations

$$
\ddot{q}=-D_{q} U(q)-\lambda D_{q} c(q), \quad c(q)=0 .
$$

As the constraint imposes $\dot{c}(q)$ on the dynamics it is certainly also true that all higher derivatives vanish identically. In particular $\ddot{c}(q)=0$, which in detail reads,

$$
0=\left\langle D_{q} c, \ddot{q}\right\rangle+\left\langle D_{q}^{2} c \dot{q}, \dot{q}\right\rangle .
$$

Now we can plug the equations of motion into the equation for $\ddot{c}$ and solve for the constraint force $-\lambda D_{q} c$. On condition that $\dot{q}=\dot{\sigma}$ is tangent to $\Sigma$ this yields

$$
-\lambda D_{q} c(\sigma)=\nu(\sigma)\left(\left\langle\nu(\sigma), D_{q} U(\sigma)\right\rangle-\langle S(\sigma) \dot{\sigma}, \dot{\sigma}\rangle\right),
$$

where $\nu=n /\|n\|$ with $n=D_{q} c$ denotes the unit normal to the constraint surface and $S=-\mathrm{d} \nu[\cdot]$ is again the matrix of the Weingarten map — but here in the mass-scaled Euclidean basis. Comparing the first term in the last equation to (2.5) we see that it basically equals $\partial_{c} U$. Indeed $S$ is the shape operator is easy to see as

$$
\mathrm{d} \nu[\cdot]=\|n\|^{-2}\left(\|n\| \mathrm{d} n[\cdot]-n\|n\|^{-1}\langle n, \mathrm{~d} n[\cdot]\rangle\right)=\|n\|^{-1} H_{\sigma} \mathrm{d} n[\cdot],
$$

which by definition is the Weingarten map. However we have to be careful since we have to read the last equation from right to left, and the identification is valid only on condition that $\dot{c}(q)=0$, that is, $\dot{q}=H_{\sigma} \dot{q}$ is tangent to the constraint surface.

\section{Appendix B. Generating random momenta for constrained HMC.}

In order to implement the HMC algorithm, we have to randomly draw a velocity or momentum vector, respectively, from the constrained canonical density $\mu_{\Sigma}$. As we have shown by means of the Poisson bracket identity (3.3), the constrained density is simply obtained from a restriction of the original canonical density to $T^{*} \Sigma$. So the easiest way to draw momenta from the constrained invariant distribution is to 
1. generate a vector $X$ according to the unconstrained canonical distribution,

2. and then apply the projection of the vector $X$ onto the constraint surface.

We shall shortly explain, why this works, and why the projected vector is properly distributed. For obvious reasons, we may restrict our attention to only the momentum part of the invariant density, and we drop the mass-scaling. Up to a factor $1 / 2$ the kinetic energy is then represented by the quadratic form $T=\left\langle M^{-1} X, X\right\rangle$, describing a hypersphere in a mass-weighted Euclidean norm. In the following we regard $X$ as a vector in $\mathbf{R}^{m}$. Suppose, we set $T=1$, which means to define a unit hypersphere $S^{m-1}$, or an isodensite in momentum space, respectively. Recall that $H_{\sigma}$ is the orthogonal projection onto the constraint distribution $\mathcal{D}(\sigma)$. Accordingly

$$
H_{\sigma}^{*}=\mathbf{1}-V_{\sigma}^{*} \quad \text { with } \quad V_{\sigma}^{*}=M \nu\left\langle M^{-1} \nu, \cdot\right\rangle
$$

is the orthogonal projector onto the constraint codistribution $\mathcal{D}^{*}(\sigma)$, where $\nu=\nu(\sigma)$ is the unit normal to $\mathcal{D}(\sigma)$. Geometrically, the constrained isodensite is the intersection of the codistribution at $\sigma \in \Sigma$ with the isodensite $I=\left\{X \in \mathbf{R}^{m} \mid T(X)=1\right\}$. Hence $H_{\sigma}^{*} \circ S^{m-1}=S^{m-2}$, and we have to identify the norm, in which $S^{m-2}$ is again a unit hypersphere - of lower dimension however. Obviously for any vector $X$ in $\mathcal{D}^{*}$, the identity $H_{\sigma}^{*} X=X$ holds, and so does it on $\mathcal{D}^{*} \cap I$. Hence, for $X^{\prime} \in \mathcal{D}^{*} \cap I$ the following relation holds:

$$
1=\left\langle H_{\sigma}^{*} X^{\prime}, M^{-1} H_{\sigma}^{*} X^{\prime}\right\rangle=\left\langle X^{\prime}, H_{\sigma}^{*} M^{-1} X^{\prime}\right\rangle,
$$

describing a degenerate unit hyperellipse in the metric which is determined by the projected inverse mass matrix $H_{\sigma}^{*} M^{-1}$. Note that $H_{\sigma}^{*}$ and $M^{-1}$ commute, and therefore we can forget about the second projector. But according to the considerations in the derivation of (3.3) this is in fact the correct covariance matrix for the constrained momentum distribution in Cartesian coordinates. It is degenerate, because the projector $H_{\sigma}^{*}$ has reduced rank $m-1$, but $X^{\prime}$ is a vector in $\mathbf{R}^{m}$. In this way the projection maintains the full dimensionality for the HMC algorithm.

\section{Appendix C. Example: Free energy computation for n-Butane.}

We illustrate how the constrained HMC algorithm works. For the short-time integration steps we use the RATTLE symplectic integrator. In order to sufficiently guarantee long-time stability, the constraint has to be satisfied exactly; this is done by means of a Newton iteration solving the nonlinear equations [38]. In order to obtain a physically meaningful result we have to remove the mass scaling first. To this end we introduce a rescaled metric

$$
\|X\|_{M^{-1}}^{2}=\left\langle X, M^{-1} X\right\rangle, X \in \mathbf{R}^{m}
$$

which renders the expression (3.2) for the force in the way that

$$
f(q)=-\|n\|_{M^{-1}}^{-1}\left(\left\langle\nu, M^{-1} D_{q} U\right\rangle-\beta^{-1} \kappa-\beta^{-1} \omega[\nu]\right), \quad q \in \Sigma
$$

with

$$
\omega[\nu]=\|n\|_{M^{-1}}^{-2}\left\langle n, M^{-2} D_{q}^{2} c \nu\right\rangle \quad \text { and } \quad \kappa=\|n\|_{M^{-1}}^{-1} \operatorname{tr}\left(M^{-2} H_{q} D_{q}^{2} c\right) .
$$

Bear in mind that the two inverse masses come into play from the second derivatives with respect to the scaled variables, and also from the momentum average. Also note 
that the normalisation of the unit normal $\nu$ is understood with respect to $\|\cdot\|_{M^{-1}}$. A rather compact representation of the force is due to [9]

$$
f(q)=-\|n\|_{M^{-1}}^{-1}\left\langle M^{-1} \nu, D_{q} U\right\rangle+\beta^{-1} \operatorname{div}\left(M^{-1} \nu\|n\|_{M^{-1}}^{-1}\right), \quad q \in \Sigma .
$$

The HMC scheme consists of the usual proposal and acceptance step, with the numerical integration using the RATTLE symplectic integrator in between. Let $\left(q_{n}, p_{n}\right)$ satisfy the constraints. Then the algorithm proceeds as follows:

1. choose a random momentum vector $\xi \in \mathbf{R}^{m}$ according to $\xi \sim \mathcal{N}(0, M)$

2. apply the projection $\xi \mapsto\left(\mathbf{1}-V_{q_{n}}^{*}\right) \xi$ onto the codistribution $\mathcal{D}^{*}\left(q_{n}\right)$

3. compute the total energy $H_{n}=H\left(q_{n}, \xi\right)$ from the Hamiltonian

4. propagate $\left(q_{n+1}, \zeta\right)=\varphi^{\tau}\left(q_{n}, \xi\right)$ using the discrete flow map $\varphi^{\tau}$

5. evaluate the total energy $H_{n+1}=H\left(q_{n+1}, \zeta\right)$ again

6. accept $q_{n+1}$ with probability $\min \left\{1, \exp \left(-\beta\left(H_{n}-H_{n+1}\right)\right)\right\}$ or reject

Note that in the last step the acceptance probability using the unconstrained Hamiltonian $H(\cdot, \cdot)$ is equal to the expression in $(4.1)$, since all arguments are vectors satisfying the constraints and thus $\mu_{\Sigma}$ equals $\mu$. The whole procedure then generates a discrete time series in $\mathbf{R}^{m}$ where the points $\left\{q_{1}, q_{2}, \ldots, q_{N}\right\}$ are distributed according to the constrained distribution $Q_{\Sigma} \propto\|n(q)\|_{M^{-1}} \exp (-\beta U(q))$. We can kill the weight in front of the exponential dividing it by the blue moon factor. With the above expressions for the partially averaged force we obtain

$$
f_{\text {mean }}\left(c^{\prime}\right)=\sum_{l=1}^{N} Z\left(q_{l}\right)^{-1} f\left(q_{l}\right) / \sum_{l=1}^{N} Z\left(q_{l}\right)^{-1},
$$

where $Z=\|n\|_{M^{-1}}$ is the usual blue moon weight. Ergodicity is guaranteed by the irreducibility and positive recurrence of the underlying Markov chain [39], such that we can compute the average by means of the time series. Once the mean force has been computed for successive values of the reaction coordinate $c^{\prime}$ the free energy can be extracted from numerical integration along the reaction coordinate.

As an illustration the figure below shows the potential of mean force for a Butane molecule. We used the united-atoms (UA) force field by Ryckaert and Bellmanns [40]. Since the UA force field contains no Lennard-Jones interaction for Butane, the free energy should be identical to the torsion potential for all temperatures. We compute the free energy in $[0,2 \pi]$ on a grid of 30 points. All data were calculated at $1 / \beta=600 \mathrm{~K}$ from a 50ps HMC trajectory with $1 f s$ step size using the RATTLE integrator, and the acceptance-rejection step every $50 \mathrm{fs}$.

Let us concludingly comment on two outstanding numerical aspects. Firstly, for very high temperatures above $T=1000 K$ the molecule may move too far away from the constraint surface in a single integration step due to eventually chosen large momenta. As a consequence the Jacobian in the Newton iteration solving for the constraint will be badly scaled and will have a poor condition number. This lack of convergence can be tackled by using very small step sizes, or by using a clever damping scheme in the iteration. Secondly, for torsion angle constraints we recommend to carefully deal with the mean curvature term; computing the partial trace of the Hessian matrix on the horizontal subspace substituting $H_{\sigma}=\mathbf{1}-V_{\sigma}$ a mass weighted Laplacian arises from the first term which is very small though it is different from zero. Thus computing the trace of the shape matrix is numerically unstable due to extinction effects while evaluating the Laplacian. Even worse, dividing the mean curvature term by $Z=\|n\|_{M^{-1}}$ which is a very small number again, amplifies the absolute error dramatically. 


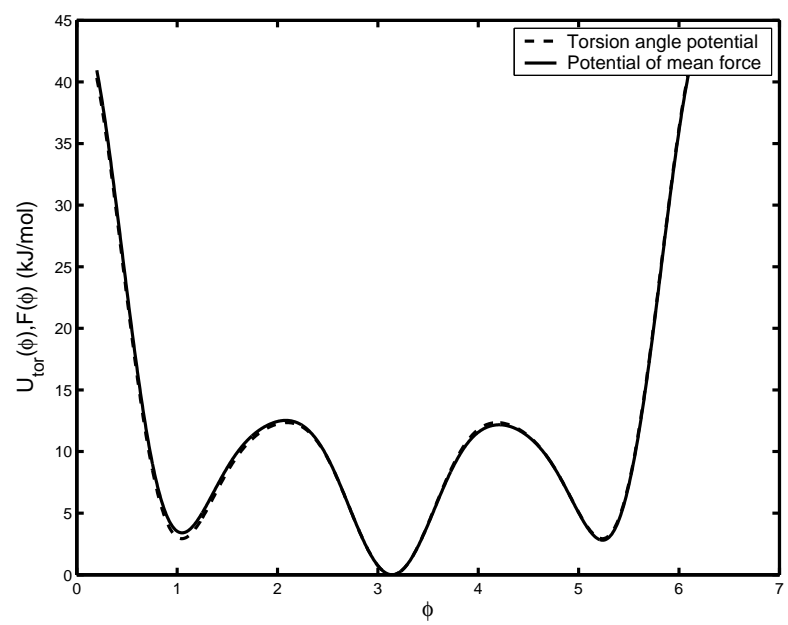

FIG. C.1. Torsion angle potential $U(\phi)$ and potential of mean force $F(\phi)$ for Butane in $U A$ representation. The data were calculated from a 50ps HMC trajectory with the provided simulation scheme at $600 \mathrm{~K}$. We suspect that the numerical error stems from computing the mean curvature $\kappa$ which is badly conditioned (see the explanation below).

\section{REFERENCES}

[1] M. Sprik and G. Ciccotti, Free energy from constrained molecular dynamics, J. Chem. Phys., 109, 18, 7737-7744, 1998.

[2] E. Darve, M. A. Wilson and A. Pohorille, Calculating free energies using a scaled-force molecular dynamics algorithm, Mol. Sim., 28, 1-2, 113-144, 2002.

[3] C. Chipot and D. A. Pearlman, Free energy calculations, the long and winding gilded road, Mol. Sim., 28, 1-2, 1-12, 2002.

[4] B. Roux, The calculation of the potential of mean force using computer simulations, Comp. Phys. Comm., 91, 275-282, 1995.

[5] J. VandeVondele and U. Rothlisberger, Efficient multidimensional free energy calculations for ab initio molecular dynamics using classical bias potentials, J. Chem. Phys., 113, 12, 4863$4868,2000$.

[6] J. G. Kirkwood, Statistical mechanics of fluid mixtures, J. Chem. Phys., 3, 300-313, 1935.

[7] T. Mülders, P. Krüger, W. Swegat and L. Schlitter, Free Energy as the potential of mean force, J. Chem. Phys., 104, 12, 4869-4870, 1996.

[8] W. K. den Otter and W. J. Briels, The Calculation of free-energy differences by constrained molecular-dynamics simulations, J. Chem. Phys., 109, 11, 4139-4146, 1998.

[9] W. E and E. Vanden-Eijnden, Metastability, conformation dynamics, and transition pathways in complex systems, Multiscale Modelling and Simulation, Springer, Berlin, 35-68, 2004.

[10] M. J. Ruiz-Montero, D. Frenkel and J. J. Brey, Efficient schemes to compute diffusive barrier crossing rates, Mol. Phys., 90, 6, 925-941, 1997.

[11] E. A. Carter, G. Ciccotti, J. T. Hynes and R. Kapral, Constrained reaction coordinate dynamics for the simulation of rare events, Chem. Phys. Lett., 156, 5, 472-477, 1989.

[12] W. G. Hoover, Canonical dynamics: Equilibrium phase-space distributions, Phys. Rev. A, 31, 3, 1695-1697, 1985.

[13] B. L. Holian and W. G. Hoover, Numerical test of the Liouville equation, Phys. Rev. A, 34, 5, 4229-4239, 1986.

[14] G. J. Martyna, Klein and M. E. Tuckerman, Nosé-Hoover chains: the canonical ensemble via continuous dynamics, J. Chem. Phys., 97, 4, 2635-2643, 1992.

[15] H. C. Andersen, Molecular dynamics simulations at constant temperature and/or pressure, J. Chem. Phys., 71, 4, 2384-2393, 1980.

[16] S. D. Bond, B. J. Leimkuhler and B. B. Laird, The Nosé-Poincaré method for constant temperature molecular dynamics, J. Comp. Phys., 151, 114-134, 1999. 
[17] A. J. vander Schaft and B. M. Maschke, On the Hamiltonian formulation of nonholonomic mechanical systems, Rep. Math. Phys., 34, 2, 225-233, 1994.

[18] R. Abraham, J. E. Marsden and T. Ratiu, Manifolds, Tensor Analysis, and Applications, Springer, New York, 1988.

[19] M. Spivak, Differential Geometry, Publish or Perish, Boston, 3, 1975.

[20] H. Rubin and P. Ungar, Motion under a strong constraining force, Comm. Pure Appl. Math., 10, 65-87, 1957

[21] F. Takens, Motion under the influence of a strong constraining force, in Global Theory of Dynamical Systems, Springer, Berlin, 99, 1980.

[22] G. Gallavotti, The Elements of Mechanics, Springer, New York, 1983.

[23] V. I. Arnold, Mathematical Methods of Classical Mechanics, Springer, New York, 1978.

[24] R. Herbst and I. Froese, Realizing holonomic constraints in classical and quantum mechanics, Comm. Math. Phys., 220, 489-535, 2001.

[25] J. E. Marsden and T. S. Ratiu, Introduction to Mechanics and Symmetry, Springer, New York, 1999.

[26] D. Frenkel and B Smit, Understanding Molecular Dynamics: From Algorithms to Applications, Academic Press, London, 2002.

[27] J. E. Marsden, R. Montgomery and T. S. Ratiu, Reduction, symmetry and phases in mechanics, Memoirs AMS, 88, 436, 1-110, 1990

[28] P. A. M. Dirac, Generalized Hamiltonian dynamics, Can. J. Math, 2, 129-148, 1950.

[29] B. Leimkuhler and S. Reich, Symplectic integration in constrained Hamiltonian systems, Math. Comp., 63, 589-605, 1994.

[30] R. I. McLachlan and C. Scovel, Equivariant constrained symplectic integration, J. Nonlinear Sci., 5, 233-256, 1995.

[31] M. E. Tuckerman, Y. Liu, G. Ciccotti and G. J. Martyna, Non-Hamiltonian molecular dynamics: Generalizing Hamiltonian phase space principles to non-Hamiltonian dynamics, J. Chem. Phys., 115, 4, 1678-1702, 2001.

[32] S. Melchionna, Constrained systems and statistical distribution, Phys Rev. E, 61, 6, 6165-6170, 2000 .

[33] R. A. Horn, Matrix Analysis, Cambridge University Press, 1987.

[34] M. Fixman, Classical statistical mechanics of constraints: a theorem and applications to polymers, PNAS, 71, 3050-3053, 1974.

[35] J. S. Liu, Monte Carlo Strategies in Scientific Computing, Springer, New York, 2001.

[36] M. P. do Carmo, Riemannian Geometry, Birkhäuser, Boston, 1992.

[37] B. A. Dubrovin, A. T. Fomenko and S. P. Novikov, Modern Geometry - Methods and Applications: Part I. The Geometry of Surfaces, Transformation Groups, and Fields, Springer, Berlin, 1984.

[38] E. Hairer, C. Lubich and G. Wanner, Geometric Numerical Integration, Springer, Berlin, 2002.

[39] P. Brémaud, Markov Chains: Gibbs Fields, Monte Carlo Simulation, and Queues, Springer, New York, 1999.

[40] J. P. Ryckart and A. Bellmanns, Molecular dynamics of liquid n-Butane near its boiling point, Chem. Phys. Lett., 30, 1, 123-125, 1975. 